\title{
Web report Critical Care Assembly Critically Appraised Topics (CCCATS) Library
}

\author{
Nicolino Ambrosino
}

Director, Pulmonary and Respiratory Intensive Care Unit, Cardio-Thoracic Department, University Hospital, Pisa, Italy

Correspondence: Nicolino Ambrosino, n.ambrosino@ao-pisa.toscana.it

Reported: 30 April 2003

Published online: 30 May 2003

This article is online at http://ccforum.com/content/7/4/327

(c) 2003 BioMed Central Ltd (Print ISSN 1364-8535; Online ISSN 1466-609X)

Website Critical Care Assembly Critically Appraised Topics Library

URL http://www.thoracic.org/criticalcare/cccat.asp

Cost Free

Keywords evidence-based medicine, intensive care

The Critical Care Assembly Critically Appraised Topics is a website that provides updates in relevant and controversial critical care topics using an evidence-based approach. In reviewing this site we browsed through the online offerings and assessed the information provided as well as the user interface. Each critically appraised topic (CAT) is structured so that the following information is included: the search strategy employed to identify significant trials in the area; a summary of each article identified, which includes a grading of the evidence, number of patients and major results; and short editorial comments. Each CAT is peer reviewed and updated as the literature warrants.

The current library of CATs includes evidence-based reviews of 14 topics, including the following: weaning protocols; prone position in acute respiratory distress syndrome; vasopressin in septic shock; continuous renal replacement therapy in sepsis; thrombolytic therapy for acute pulmonary embolism (updated); glucose control in the critically ill; maximizing oxygen delivery in critically ill patients; and low tidal volume ventilation in acute respiratory distress syndrome.

An example of the first topic is 'Noninvasive ventilation in hypoxemic respiratory failure'. According to the information provided on the website, PubMed and Uptodate were searched for articles published after 1990, using specific clinical queries such as 'noninvasive ventilation' and 'acute respiratory failure'. Seven trials were critically evaluated and their results summarized. Finally, the author of the CAT summarizes the study results and provided recommendations. This CAT was last updated in August 2001.
This online resource is intended for intensivists and respiratory physicians devoted to critical care. It provides a brief summary of topics that are extremely important and helps intensivists to identify the most recent literature for each topic. Other positives include a direct link to PubMed, the abstract of each trial, and a brief summary of each trial including design and major results. In general, the conclusions of each of these reviews are reasonable and objective. Finally, there is an invitation for the public to submit CATs. There is unlimited access to the site, no particular browser or any special software is required, and it is easy to navigate. The owner is the American Thoracic Society - a reputable scientific society.

Limitations of this site include the following. At present, there are only 14 CATs, most of which have been written by one individual. In addition, at the moment, only two topics have been updated. Finally, it would be helpful to include some CATs that are relevant to pediatrics.

\section{Best feature}

All of the topics reviewed are important, currently 'hot' topics, and the reviews will help intensivists to identify the most recent literature for each reference.

\section{Worse feature}

The reviews are very brief, and therefore for most intensivists they may not serve as complete, thorough reviews of each topic, but as an introduction/guide to the relevant trials to read.

\section{Wish list}

Other topics of interest to intensivists would be welcome. 


\section{Other links}

Southwestern Ontario Regional Academic Health Science Network (SORAHSN) - http://ahsn.lhsc.on.ca/cat/

Evidence-based Decision Making http://www.evidencebased.net/

Both sites provide the reader with access to a series of critically appraised articles tailored toward the health care decision maker. The appraisals use the format of the JAMA series Critical Appraisal Guides, which can be found online at the Canadian Centres for Health Evidence website (http://www.cche.net/principles/main.asp). Both sites also accept submissions.

\section{Competing interests}

None declared. 